\title{
Dynamic Function of Damage and Its Implications
}

\author{
Y.L. Bai ${ }^{1}$, M.F. Xia ${ }^{1,2}$, F.J. Ke ${ }^{1,3}$ and J. Bai ${ }^{1}$ \\ ${ }^{1}$ Laboratory for Non-Linear Mechanics of Continuous Media, Institute of Mechanics, \\ Chinese Academy of Science, Beijing 100080, China \\ ${ }^{2}$ Department of Physics, Peking University, Beijing 100871, China \\ ${ }^{3}$ Department of Applied Physics, Beijing University of Aeronautics and Astronautics, \\ Beijing 100083, China
}

Keywords: Dynamic Function of Damage (DFD), Damage Evolution Rate, Microdamage, Damage Moment, Compound Damage, Damage Localization

\begin{abstract}
Dynamic function of damage is the key to the problem of damage evolution of solids. In order to understand it, one must understand its mesoscopic mechanisms and macroscopic formulation. In terms of evolution equation of microdamage and damage moment, a dynamic function of damage is strictly defined. The mesoscopic mechanism underlying self-closed damage evolution law is investigated by means of double damage moments. Numerical results of damage evolution demonstrate some common features for various microdamage dynamics. Then, the dynamic function of damage is applied to inhomogeneous damage field. In this case, damage evolution rate is no longer equal to the dynamic function of damage. It is found that the criterion for damage localization is closely related to compound damage. Finally, an inversion of damage evolution to the dynamic function of damage is proposed.
\end{abstract}

\section{INTRODUCTION}

The key to continuum damage mechanics introduced by Kachanov [1] is its evolution law

$$
\dot{D}=\frac{K}{(1-D)^{\gamma}}
$$

where $\gamma$ is an undetermined parameter and $K$ is a stress-dependent function $K(\sigma)$. The nominal stress in damaged solid $\sigma$ is assumed to be related to the stress in matrix $\sigma_{s}$,

$$
\sigma=\sigma_{s}(1-D) \text {. }
$$

Actually, the continuum damage $D$ is a collective response of microdamages. In this sense, the damage evolution law (1) means that damage evolution rate depends on the damage itself as well as stress. Then, the damage can be included in constitutive equation as an internal variable. This is a macroscopically self-closed formulation, regardless any microscopic mechanisms. Lemaitre [2], Chaboche[3] and Hayhurst et.al.[4] proposed a variety of data fittings of the evolution law of damage. Clearly, this type of damage evolution law can be easily applied to engineering. However, when one intends to associate the damage evolution with material structures, he has to understand the relationship between its underlying mesoscopic mechanisms and macroscopical formulation. 
Considering the population of microdamages, we have established a fundamental equation of microdamage in the light of statistical mesoscopic damage mechanics $[5,6]$. This is the evolution equation of microdamages in phase space,

$$
\frac{\partial}{\partial t}+\sum_{i=1}^{I} \frac{\partial}{\partial} \frac{\left(n P_{i}\right)}{p_{i}}=n_{N}-n_{A},
$$

where $n$ is the number density of microdamage, $t$ is generalized time, like actual time, nominal strain, cycles, etc., $P_{i}=\dot{p}_{i}$ are the rates of variables $p_{i}, n_{N}$ and $n_{A}$ are nucleation and annihilation rates of number density of microdamages, respectively. The latter two terms may also result from the coalescence of microdamages.

We are facing four essential problems on damage evolution. They are all closely related to dynamic function of damage.

(1) What are the relations and differences between damage evolution rate and dynamic function of damage.

(2) What is the mesoscopic mechanism of macroscopically closed form of damage evolution law.

(3) What are the common features of dynamic function of damage for various mesoscopic mechanisms of microdamage.

(4) What feature of dynamic function of damage governs damage localization.

In order to solve these four problems, one has to deal with three independent variables in phase space, i.e. the current size of microdamage $c$, the initial size of microdamage $c_{0}$ and the spatial coordinates $\mathbf{x}$. Then, we should examine three different phase spaces, see Table 1.

Table 1

\begin{tabular}{|c|c|c|c|c|}
\hline case & phase space, $p_{i}$ & rate, $\quad \dot{p}_{i}$ & & remarks \\
\hline 1 & $\{c\}$ & $\dot{c}=A$ & & one-dimensional \\
\hline 2 & $\left\{c, c_{0}\right\}$ & $\dot{c}=V$ & $\dot{c}_{0} \equiv 0$ & two-dimensional \\
\hline 3 & $\{c, \mathbf{x}\}$ & $\dot{\mathcal{c}}=A$ & $\dot{\mathbf{x}}=\mathbf{v}$ & damage field \\
\hline
\end{tabular}

\section{DEFINITION OF DYNAMIC FUNCTION OF DAMAGE}

Firstly, let us examine an element of material containing distributed microdamage. Suppose $\tau$ denote the average failure volume of a microdamage with current size $c$. According to the implication of damage $D$ in expression (2), the damage should be defined as follows,

$$
D=\int_{0}^{\infty} n(t, c, \mathbf{x}) \tau d c
$$

for spherical microdamage $\tau=\frac{\pi}{6} c^{3}$. For homogeneously distributed microdamage, we can ignore its dependence on spatial coordinates $\mathbf{x}$. When multiplying the one-dimensional form of equation (3) by $\tau$ and then integrating the equation,

$$
\int_{0}^{\infty} d c \cdot \tau \cdot \quad\left[\frac{\partial}{\partial} \frac{n}{t}+\frac{\partial}{\partial} \frac{(n A)}{c}=n_{N}-n_{A}\right]
$$


with boundary conditions

$$
n A \tau \rightarrow 0 \text {, when } \quad c \rightarrow 0 \text { and } c \rightarrow \infty,
$$

we obtain the equation of damage evolution

$$
\frac{\partial D}{\partial t}=f
$$

Here, for clarification, we call equation (7) the evolution law of damage and $\dot{D}=\frac{\partial D}{\partial t}$ the damage evolution rate for the time being.

$$
f=\int_{0}^{\infty}\left(n_{N}-n_{A}\right) \tau d c+\int_{0}^{\infty} n A \tau^{\prime} d c
$$

is defined as dynamic function of damage, where $\tau^{\prime}=\frac{\partial \tau}{\partial c}$. In the concerned case, the dynamic function of damage $f$ is equal to the damage evolution rate. Then, damage can be assumed as an internal variable in constitutive equation.

More importantly, The function(DFD) $f$ is uniquely dependent on the mesoscopic dynamics of microdamage. One can notice that the function consists of two parts. The first is dependent on nucleation and annihilation of microdamage. At early stage of damage evolution, annihilation is less significant, so this term is simply dependent on nucleation. Whereas, the second term is dependent on the growth of microdamage as well as the number density of microdamage. So, the feedback from damage to the dynamic function of damage is directly included in the second term.

\section{CLOSED FORM OF DAMAGE EVOLUTION LAW AND DOUBLE DAMAGE MOMENTS}

More generally, damage $D$ is equivalent to damage moments. For example, in one-dimensional phase space and $\tau=\alpha c^{j}$,

$$
D_{j}=\alpha \int_{0}^{\alpha} c^{j} n(t, c) d c \text {. }
$$

where $\alpha$ is a shape factor. Moreover, we have double damage moments in two dimensional phase space, $\left\{c, c_{o}\right\}[6]$,

$$
D_{i, j}=\alpha \int_{0}^{\infty} d c_{o} \int_{c_{o}}^{\infty} d c c_{o}^{i} c^{j} n_{o}\left(t, c, c_{o}\right) .
$$

A self-closed formulation of damage evolution law has been investigated for the case with linear growth law $A \sim c$ in one dimensional phase space [7]. But most experimental and theoretical studies have shown that the growth law of microdamage is not only dependent on the current size of microdamage but also on its initial size [8-11]. For simplicity, here we adopt the following linear growth law [11],

$$
V=\frac{V_{s}\left(c-c_{0}\right)}{c^{*}}
$$

where $V_{s}$ is a stress dependent function and $c^{*}$ is a length scale of microdamage. We have already revealed that damage evolution is essentially due to the movement of the front of microdamage in two dimensional phase space $\left\{c, c_{0}\right\}$ [12]. Now, we demonstrate that a linear growth law (11) in two-dimensional phase space can also lead to a closed form of damage evolution law under constant stress, as that in one dimensional phase space.

The evolution of double damage moments can be derived as Eq.(5) [6], 


$$
\begin{gathered}
\dot{D}_{i, j}=D_{N i+j}+D_{G i, j}, \\
D_{N i+j}=\alpha \int_{0}^{\infty} n_{N}(c ; \sigma) c^{i+j} d c, \\
D_{G i, j}=\alpha j \int_{0}^{\infty} d c_{o} \int_{c_{o}}^{\infty} d c c_{o}^{i} c^{j-1} V\left(c, c_{o} ; \sigma\right) n_{o}\left(t, c, c_{o}\right) .
\end{gathered}
$$

Here we have ignored the term of annihilation. After substituting the linear growth law (11) into Eqs. (12-14), the double damage moment rate $\dot{D}_{i j}$ is expressed by

$$
\dot{D}_{i, j}=D_{N i+j}+\alpha j V_{s}\left(D_{i, j}-D_{i+1, j-1}\right) / c^{*} .
$$

Actually, Eq.(15) is macroscopically closed recurrently. For example, the double damage moment with $i=0$ and $j=3$, correspond to volumetric fraction of damage. Then, recurrently closed equations of damage rate $\dot{D}=\dot{D}_{0,3}=f$ are

$$
\begin{aligned}
f=\dot{D}_{0,3} & =D_{N 3}+3 \alpha V_{s}\left(D_{0,3}-D_{1,2}\right) / c^{*} \\
\dot{D}_{1,2} & =D_{N 3}+2 \alpha V_{s}\left(D_{1,2}-D_{2,1}\right) / c^{*} \\
\dot{D}_{2,1} & =D_{N 3}+\alpha V_{s}\left(D_{2,1}-D_{3,0}\right) / c^{*} \\
\dot{D}_{3,0} & =D_{N 3} .
\end{aligned}
$$

This implies that for linear growth law of microdamage either in one or two dimensional phase space, the damage rate $\dot{D}$ can always be expressed in a macroscopically closed form. Then, the left problem is whether closed form of damage evolution is an appropriate approximation for various mesoscopic mechanisms of microdamage.

\section{NUMERICAL RESULTS OF DAMAGE EVOLUTION}

We have the system of equations of damage evolution in two dimensional phase space

$$
\begin{aligned}
& \frac{\partial n_{o}}{\partial t}+\frac{\partial\left(n_{o} V\right)}{\partial c}=n_{N} \delta\left(c-c_{o}\right) \\
& D=D^{*} \int_{0}^{o} d c \int_{0}^{c} c^{3} n d c_{o}, \\
& \sigma=\frac{\sigma_{0}}{1-D}
\end{aligned}
$$

In the numerical study, we assumed the third damage moment $D_{3}$, i.e. the volumetric fraction of damage, as the continuum damage $D$ and ignored the term of annihilation. Also, all variables in this section are nondimensionalized with following characteristic parameters, $c^{*}, V^{*}, n_{N}^{*}$ and $\sigma^{*}$. Then, $D^{*}=\alpha n_{N}{ }^{*} c^{* 5} / V^{*}$ is a dimensionless parameter. There are two undetermined characteristic scales, time scale $t_{k}$ and scale of number density of microdamage $n_{k}$. To balance the three terms of Eq. (17), we obtained $t_{k}=c^{*} / V^{*}$ and $n_{k}=n_{N} c^{*} / V^{*}$. The number density of microdamage $n_{o}$ in two-dimensional space is nondimensionalized with the characteristic scale $n_{k} / c^{*}=n_{N} * / V^{*}$.

The corresponding initial and boundary conditions are

$$
\begin{aligned}
& n_{o}\left(0, c, c_{o}\right)=0 \\
& n_{o}\left(t, c, c_{0}\right)=0 \quad \text { when } c \leq c_{o} .
\end{aligned}
$$


The characteristics of equation (17) are

$$
\begin{aligned}
& \frac{d c_{o}}{d t}=0, \\
& \frac{d c}{d t}=V, \\
& \frac{d n_{o}}{d t}=n_{N} \delta\left(c-c_{o}\right)-\left(\frac{\partial}{\partial} \frac{V}{c}\right) n_{0} .
\end{aligned}
$$

Owing to successive nucleation of microdamages, the initial conditions of the characteristics are

$$
\begin{array}{ll}
c_{o}=c_{o} & \text { when } t=0, \\
c=c_{o} & \text { when } t=t_{0}, \\
n_{o}\left(t, c, c_{o}\right)=0 & \text { when } 0 \leq t \leq t_{0}
\end{array}
$$

where $t_{0}$ is the nucleation time of microdamage with current size $c$ at time $t$.

Now, let us evaluate the intrinsic governing parameter, $D^{*}=\alpha n_{N} c^{*^{5}} / V^{*}$. For spherical failure zone of microdamage, the geometrical factor $\alpha=\pi / 6$. The combination of $n_{N} * c^{*} / V^{*}$ denotes the characteristic volumetric fraction of damage. From our impact results, the typical values of the characteristic size and growth rate of microdamage are $c^{*} \approx 4 \cdot 10^{-6} \mathrm{~m}$ and $V^{*} \approx 10 \mathrm{~m} / \mathrm{s}$, respectively $[11,13]$. The nucleation rate of number density of microdamage on sectional surface is about $10^{3} /\left(\mathrm{mm}^{2} \cdot \mu \mathrm{m} \cdot \mu \mathrm{s}\right)$. Then the transformation from sectional to volumetric count gives a nucleation rate of number density of microdamage in volume $10^{13.5} /\left(\mathrm{m}^{3} \cdot \mu \mathrm{m} \cdot \mu \mathrm{s}\right) \sim 10^{25.5} /\left(\mathrm{m}^{3} \cdot \mathrm{m} \cdot \mathrm{s}\right)$. Therefore, $D^{*}$ is in the order of $10^{25.5} \cdot\left(4 \cdot 10^{-6}\right)^{5} / 10 \sim 10^{-2.5}$. In our calculation we choose $D^{*}=0.001$.

The two mesoscopic dynamic laws, nucleation and growth rates of microdamage, according to the experimental results, were assumed as the following dimensionless forms

$$
\begin{aligned}
& n_{N}=g(\sigma-1) c \exp (-c), \\
& V=g(\sigma-1) c^{v_{1}}\left(c-c_{o}\right)^{v_{2}} .
\end{aligned}
$$

Table 2 shows three types of growth law. Additionally, we adopted the same linear function of stress $g$ in both nucleation and growth rates in calculation.

Table 2: Three types of growth rate of microdamage

\begin{tabular}{lllll}
\hline 1 & $v_{1}=1$ & $v_{2}=0$ & $V=g(\sigma-1) c$ & Curran et al. [10] \\
2 & $v_{1}=-1$ & $v_{2}=1$ & $V=g(\sigma-1)\left(1-c_{d} c\right)$ & Berry [9] \\
3 & $v_{1}=0$ & $v_{2}=1$ & $V=g(\sigma-1)\left(c-c_{o}\right)$ & Han et al. [11] \\
\hline
\end{tabular}

Both finite difference scheme and characteristics give quite close results, showing the reliability of the numerical simulation.

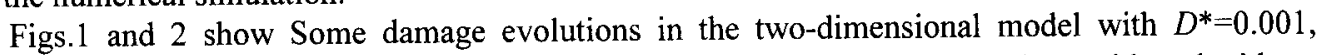
$\sigma_{o}=1.25, v_{1}=0$ and $v_{2}=0.5$. Fig. 1 stresses the comparison of damage evolutions with and without stress feedback. On the other hand, Fig. 2 demonstrates damage evolutions with the same $v_{1}=0$ but different exponents $v_{2}$. The effect of growth rate on damage evolution appears stronger than that of stress feedback. Moreover, the numerical results indicate that the increase of damage evolution rate exhibits a little concave with increasing damage $D$. Roughly speaking, the nucleation rate governs the initial damage rate, whilst the growth rate and stress feedback control the concavity. This feature has been found in experimental results, such as [4]. The common feature might be the basis of approximate closed forms of damage evolution law in practice, like Kachanov's law. 


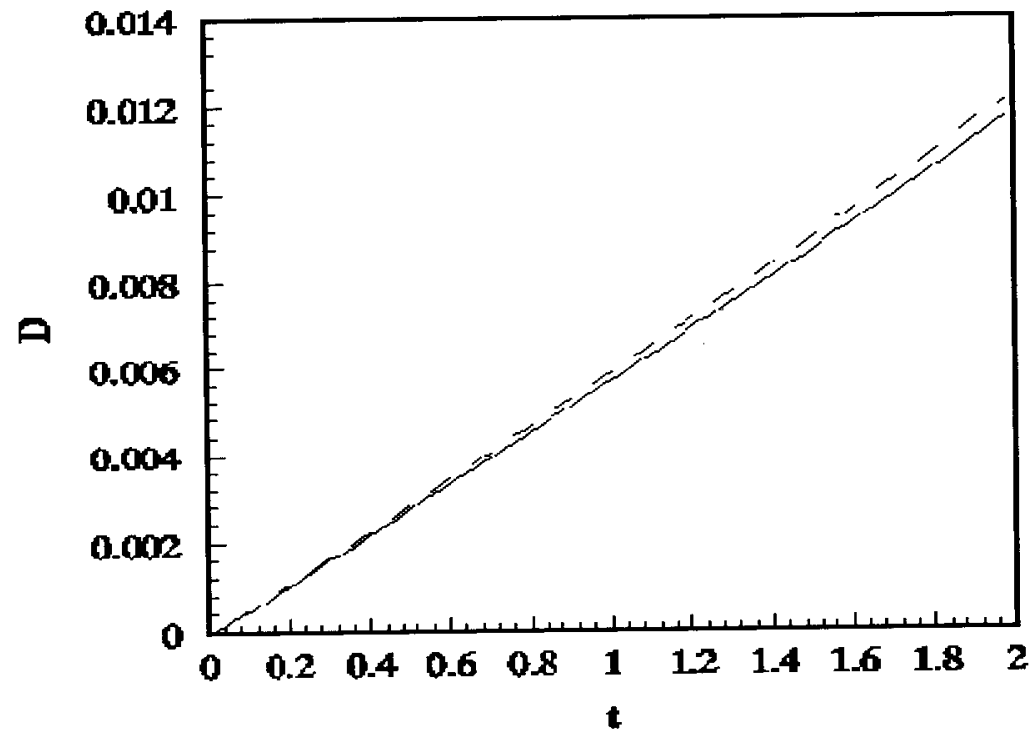

Fig. 1 Damage evolutions in two-dimensional model with (upper curve) and without (lower curve) stress feedback, $v_{2}=0.5$.

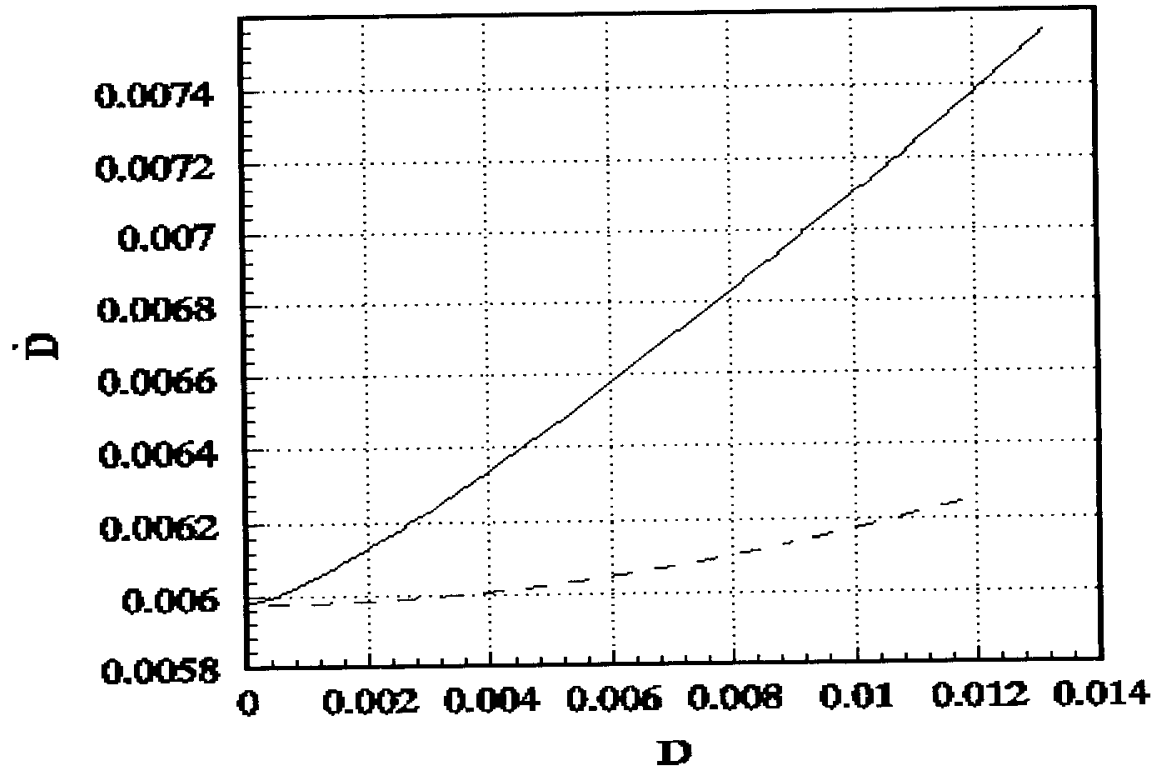

Fig. 2 Damage evolutions in two-dimensional model with different growth rates, $v_{2}=0.2$ (upper curve) and $v_{2}=0.5$ (lower curve). 


\section{DYNAMIC FUNCTION OF DAMAGE IN DAMAGE FIELD}

In previous sections, we adopted the assumption of homogeneous damage, then damage becomes an internal variable in constitutive equation. As a matter of fact, the damaged material element should be examined in a damage and deformation field [14]. This viewpoint is equivalent to the examination of damage in phase space $\{c, \mathbf{x}\}$. Then, the fundamental equation of damage evolution (3) becomes

$$
\frac{\partial D}{\partial t}+\nabla \cdot(D \mathbf{v})=f
$$

In damage mechanics, damage $D$ is defined in Eulerian coordinates, as formula (1) and (4). But as a rate of an internal variable, the partial derivative should be defined in Lagrangian coordinates in damage field, $\dot{D}=\frac{\partial D}{\partial T}$, rather than $\frac{\partial D}{\partial t}$. Transformation of Eq.(30) into Lagrangian coordinates leads to

$$
\frac{\partial D}{\partial T}+D \nabla \cdot \mathbf{v}=f \quad \text { or } \quad \frac{\partial D}{\partial T}=f-D \nabla \cdot \mathbf{v} .
$$

Therefore, unlike the equality of the derivative $\frac{\partial D}{\partial t}$ and the dynamic function of damage $f$ derived in Section 2, there is a difference between the two. This can be shown more clearly in onedimensional strain state,

$$
\frac{\partial D}{\partial T}=f-D \dot{\theta}
$$

where $\theta=\ln \left(\rho_{0} / \rho\right)$ is natural strain, $\rho_{0}$ and $\rho$ are initial and current density respectively. The second term on the right is due to the rate of dilatation. Fig. 3 shows the difference of damage evolutions with and without the dilatation rate under constant dragging velocity.

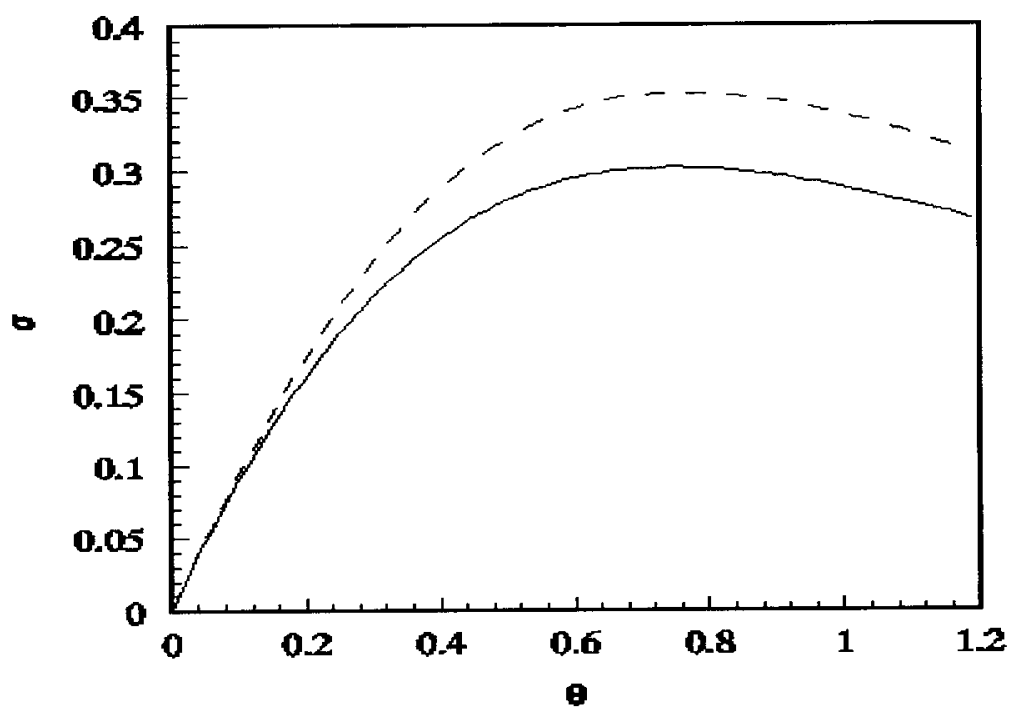

Fig.3 The difference of dimensionless stress-strain curves with (upper curve) and without (lower curve) dilatation rate term in (32). In the calculation, matrix is linear elastic and dimensionless DFD $f=\left(A+B D^{m}\right) \sigma, A=1, B=0$ and $m=2$. 


\section{COMPOUND DAMAGE AND DAMAGE LOCALISATION}

Compound damage evolution is defined as $\dot{D}=\dot{D}(D, \sigma)$ by Davison and Stevens [15], in comparison with simple damage accumulation $\dot{D}=\dot{D}(\sigma)$. As they pointed out, "the ease with which damage is accumulated depends on the amount of damage already present". The concavity in damage evolution rate shown in Section 4 reflects the compound damage. As a material property, the level of compound is better defined by the partial differentiation of DFD, namely $f_{D}=\frac{\partial f}{\partial D}$, if $f=f(D, \sigma)$. Then, what does the compound damage exactly imply in damage evolution? We have found that the ratio of the compound damage $f_{D}$ over corresponding intercept $f / D$ is critical for damage localization. Here, we outline the reasoning briefly [14].

The inhomogeneity of damage field can be defined by $\left(\frac{\partial D}{\partial Y}\right) / D$. Clearly, one can presume that the positive differentiation of the damage inhomogeneity with respect to time signifies damage localization,

$$
\left(\frac{\partial\left(\frac{\partial D}{\partial Y}\right)}{\partial T}\right) /\left(\frac{\partial D}{\partial Y}\right)>\left(\frac{\partial D}{\partial T}\right) / D .
$$

After differentiation of (32) with respect to spatial coordinate $Y$ and some manipulations, (33) can be expressed by

$$
f_{D}+\left(f_{\sigma} \frac{\partial \sigma}{\partial Y}-D \frac{\partial \dot{\theta}}{\partial Y}\right) /\left(\frac{\partial D}{\partial Y}\right)>f / D
$$

For quasistatic deformation, stress keeps uniform according to momentum conservation. Then the second term becomes zero. Moreover, for small deformation, the third term is smaller than others. Therefore, an approximate but practical criterion for damage localization is

$$
f_{D}>f / D \text {. }
$$

Fig.4 shows the criterion for damage localization schematically. Fig. 5 gives an example of damage localization.

\section{INVERSION OF DAMAGE EVOLUTION TO DYNAMIC FUNCTION OF DAMAGE}

Dynamic function of damage is the core in damage evolution, but this function deeply roots in the mesoscopic mechanisms of damage evolution. Then, how to determine the function in practice?

Certainly, the most reasonable approach to it is based on mechanical analysis of the mesoscopic mechanism, like Cocks and Ashby did [16]. But, this is not suitable for processes with multimechanisms. Moreover, these processes are the most in practice. So, from engineering point of view, we need some operational approach to it. In the light of the damage field equation, we are developing an inverse method to cope with the problem.

Define damage in unit mass $G=D / \rho$. Then, Eq.(32) can be rewritten as

$$
f=\rho \frac{\partial G}{\partial T}
$$

When the evolutions of stress, strain and damage, $\sigma(T), \theta(T)$ and $D(T)$, have been measured in practice or calculated in simulation, one can easily derive the evolutions of damage $G(T)$ as well as 


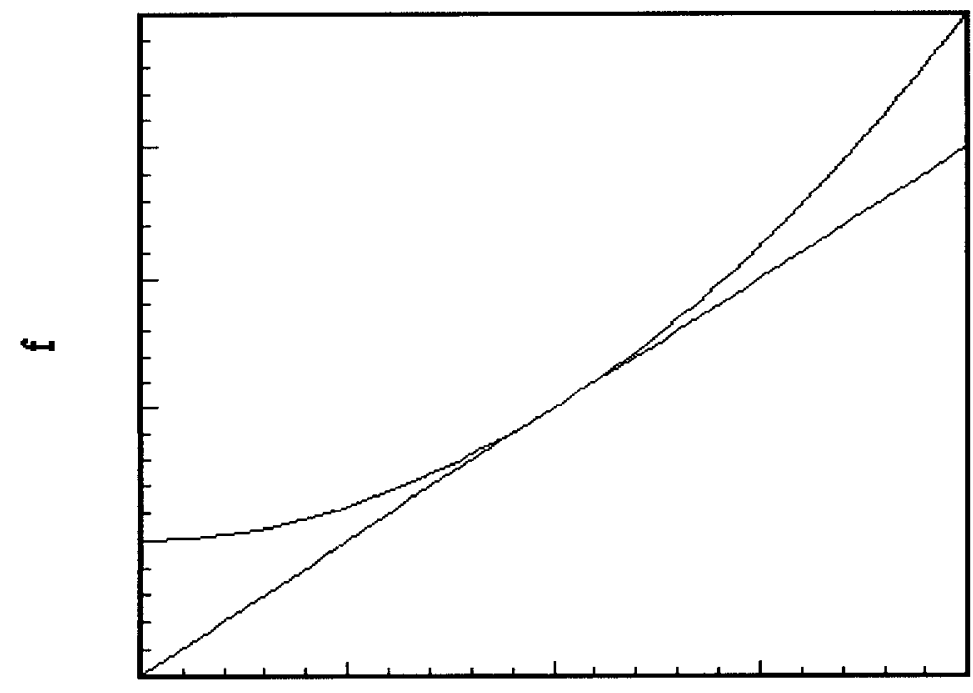

$\mathbf{D}$

Fig. 4 A schematic of criterion for damage localization.

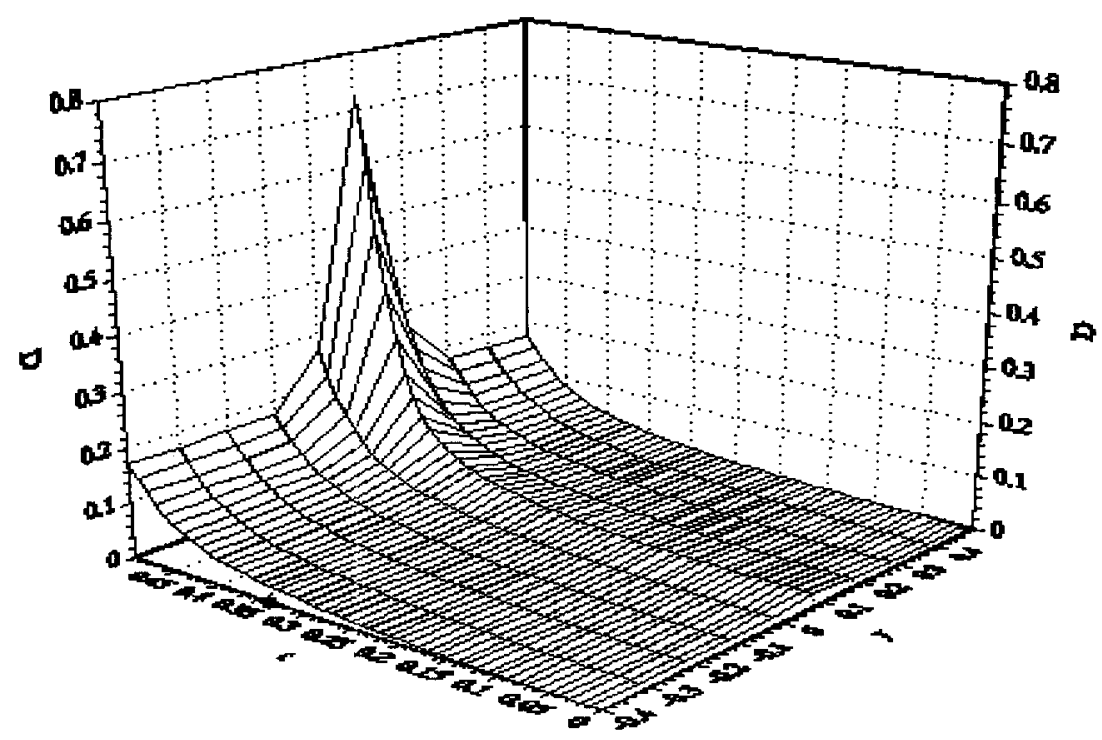

Fig. 5 An example of damage field evolution showing damage localization. In the calculation, matrix is linear elastic, dimensionless DFD $f=\left(A+B D^{m}\right) \sigma, A=0.5, B=500$ and $m=2$ and initial inhomogeneity of damage is $D_{0}=0.01 \exp \left(-Y^{2}\right)$. Critical damage given by(35) is $\sqrt{ }(A / B) \sim 0.0316$, corresponding to dimensionless time 0.34 (marked with $\bullet$ on $t$ axis). 
the dynamic function of damage $f(T)$, according to (36). Now, we choose a set of samples with different initial damage $D_{0}$ from the same material. Then all the evolutions become binary functions, like $\sigma\left(T, D_{0}\right), D\left(T, D_{0}\right)$ and $f\left(T, D_{0}\right)$. After transformation of the independent variables $T$ and $D_{0}$ into $\sigma$ and $D$, the dynamic function of damage $f$ can be expressed as binary function $f(\sigma, D)$. This is the required dynamic function of damage.

\section{Summary}

1. A dynamic function of damage is strictly defined, in terms of evolution equation of microdamage and damage moment.

2. Self-closed damage evolution law is investigated by means of double damage moments.

3. Numerical results of damage evolution demonstrate common features for various microdamage dynamics.

4. In inhomogeneous damage field, damage evolution rate is no longer equal to the dynamic function of damage.

5. A criterion for damage localization is found to be closely related to compound damage.

6. An inversion of damage evolution to the dynamic function of damage is proposed.

\section{Acknowledgments}

This work is supported by the National Natural Science Foundation of China and the Chinese Academy of Sciences.

\section{REFERENCES}

1. L.M. Kachanov, Introduction to Continuum Damage Mechanics, Martinus Nijhoff, The Netherlands (1986).

2. J. Lemaitre and A. Plumtree, ASME Trans., J. Engng. Matl. \& Tech., 1979 (101) 284-292

3. J. L. Chaboche, Trans. J. Appl. Mech., 1988 (55) 59-64, 65-72

4. D.R. Hayhurst, P.R. Dimmer and C.J. Morrison, Phil. Trans. R. Soc.. London, 1984( A311) 103-129

5. Y.L. Bai, F.J. Ke, and M.F. Xia, Acta Mechanica Sinica, 7(1991) 59-66.

6. M.F. Xia, W.S. Han, F.J. Ke and Y.L Bai, Advances in Mechanics, 25 (1995) 1 - 40.

7. Y.L. Bai, M.F.Xia, F.J. Ke, W.S. Han and B. Fang, Int. J. Fracture, 78 (1996) 331-334.

8. N.F. Mott, Engineering, 165 (1948) 16

9. J.P. Berry, J. Mech. Phys. Solids, 8 (1960), 194-206 and 207-216.

10. D.R. Curran, L. Seaman and D.A. Shockey, Physics Reports, 147 (1987) 253- 388.

11. W.S. Han, L.T. Shen and Y.L. Bai, Int. J. Solids and Structures, (1997) to be published.

12. Y.L. Bai, W.S. Han and J. Bai, ASTM STP 1315 (1997)

13. Y.L. Bai, Z. Ling, L.M. Luo, and F.J. Ke, ASME Trans., J. Appl. Mech., 59(1992) 622-627.

14. Y.L. Bai, M.F.Xia, F.J. Ke and H.L. Li, submitted to IUTAM Symp. Reology of Bodies with Defects, Sept.1997, Beijing.

15. L. Davison and A.L Stevens, J. Appl. Phys., 43 (1972) 988-994.

16. A.C.F. Cocks and M.F. Ashby, Progress in Material Science, 27(1981) 189-244. 


\section{Fracture and Strength of Solids III}

10.4028/www.scientific.net/KEM.145-149

\section{Dynamic Function of Damage and Its Implications}

10.4028/www.scientific.net/KEM.145-149.411 